\title{
Measuring the Ease of Communication in Bipartite Social Endorsement Networks
}

\author{
A Proxy to Study the Dynamics of Political Polarization
}

\author{
Morteza Shahrezaye \\ Bavarian School of Public Policy \\ Munich, Bavaria \\ morteza.shahrezaye@tum.de \\ Juan Carlos Medina Serrano \\ Bavarian School of Public Policy \\ Munich, Bavaria \\ juan.medina@tum.de
}

\author{
Orestis Papakyriakopoulos \\ Bavarian School of Public Policy \\ Munich, Bavaria \\ orestis.papakyriakopoulos@tum.de \\ Simon Hegelich \\ Bavarian School of Public Policy \\ Munich, Bavaria \\ simon.hegelich@hfp.tum.de
}

\begin{abstract}
In this work, complex weighted bipartite social networks are developed to efficiently analyze, project and extract network knowledge. Specifically, to assess the overall ease of communication between the different network sub-clusters, a proper projection and measurement method is developed in which the defined measurement is a function of the network structure and preserves maximum relevant information. Using simulations, it is shown how the introduced measurement correlates with the concept of political polarization, after which the proposed method is applied to Facebook networks to demonstrate its ability to capture the polarization dynamics over time. The method successfully captured the increasing political polarization between the Alternative für Deutschland's (AfD) supporters and the supporters of other political parties, which is in line with previous studies on the rise of the AfD in Germany's political sphere.
\end{abstract}

\section{CCS CONCEPTS}

- Networks $\rightarrow$ Social media networks; Network simulations; • Human-centered computing $\rightarrow$ Social network analysis; Social networking sites; $\bullet$ General and reference $\rightarrow$ Metrics; Estimation;

\section{KEYWORDS}

Social media networks, Bipartite network projection, Network simulations, Political polarization, Political discourse

ACM Reference format:

Morteza Shahrezaye, Orestis Papakyriakopoulos, Juan Carlos Medina Ser-rano, and Simon Hegelich. 2019. Measuring the Ease of

Communication in Bipartite Social Endorsement Networks. In

Proceedings of International Conference on Social Media and Society,

Toronto, ON, Canada, July 19-21, 2019 (SMSociety '19), 8 pages. https://doi.org/10.1145/3328529.3328556

Permission to make digital or hard copies of all or part of this work for personal or classroom use is granted without fee provided that copies are not made or distributed for profit or commercial advantage and that copies bear this notice and the full citation on the first page. Copyrights for components of this work owned by others than ACM must be honored. Abstracting with credit is permitted. To copy otherwise, or republish, to post on servers or to redistribute to lists, requires prior specific permission and/or a fee. Request permissions from permissions@acm.org.

SMSociety '19, July 19-21, 2019, Toronto, ON, Canada

(c) 2019 Association for Computing Machinery.

ACM ISBN 978-1-4503-6651-9/19/07 . \$15.00

https://doi.org/10.1145/3328529.3328556

\section{INTRODUCTION}

\subsection{Social Networks}

Following of the uptake in social media services, social scientists have been presented with significant new challenges and opportunities. The generation of huge data sets, which record the interactions of millions of users, has dramatically changed the quantitative models, research style and empirical methods that social scientists use. This renaissance requires social scientists to adapt to new quantitative methods [27].

Network analysis theory can now provide the theory and tools required for social scientists to model, study and generate knowledge from the complex interactions of millions of social media users on services such as Facebook and Twitter. However, social networks can be complex unlike most biological, technological, and other real-life networks that often have disassortative mixing or negative correlations with neighboring vertices, social networks mostly show assortative mixing or positive degree correlations with the neighboring vertices. A second distinctive feature of social networks is their topology. While non-social networks generally have no significant local clustering compared to random networks with similar degrees of distribution, social networks have been found to have significant clustering [40].

These two special social network features emerge at the time of the network formation; that is, sub-communities and assortative mixing are formed while the whole network emerges. These features emerge because of many reasons such as technological design or cognitive biases. Geschke et al. [18] used agent-based modeling to show that sub-communities formed even in the absence of technological filters. Therefore, any effort to study social networks needs to consider that these networks have special features that cannot be ignored.

This paper focused on a special type of social network. While most social and non-social networks are one-mode networks, some are two-mode or bipartite networks. While one-mode networks 
have only one type of vertices, bipartite networks have two different types of vertices and each edge is between a vertices pair of different types. For example, a friendship network is a one-mode network in which each edge between two vertices indicates that the corresponding users are friends. However, Facebook posts are part of a bipartite network, in which each edge indicates a user who has commented on the corresponding Facebook post. Both types of the mentioned networks might have weighted edges that measure the strength of the edge, or binary simple edges which only shows an unweighted connection. Bi-partite networks have been analyzed in a wide variety of different contexts, such as sports activity networks [42], actors networks [41], economics and finance networks [8], online file sharing networks [20,30] and scientific authoring networks $[38,51]$.

Because bipartite networks are more complex to study, they require different tools than studies on simple one-mode networks $[4,36,58,60]$. Studying bipartite networks requires either projecting the network to a one-mode network or developing the proper measurements applicable to the bipartite case [12]. The result of projecting a bipartite network to a one-mode network is a binary or weighted one-mode network, which could lead to the deletion of some important information [44, 61]; for example, the global and local clustering coefficients on bipartite networks differ significantly from the counterpart values in corresponding projected networks [43].

This paper argues that the usual projection methods lose a great deal of information because they do not account for the existing assortativity and the network clustering within social networks. Therefore, in this paper, first a new projection method is suggested for weighted bipartite social networks that is able to preserve the relevant information from the initial network. Afterwards, methods applicable to the resulting simple one-mode networks are employed to generate knowledge from the projected networks. The proposed method is used to demonstrate that these methods could be used as proxy measurements for monitoring political polarization dynamics, and a mathematical method is developed to study this important social and political process. Because of the rise in online social networks, political polarization has become a key research topic in social sciences; therefore, this study contributes to research in these areas and could be used to understand the tenor of a particular development.

\subsection{Political Polarization}

From well-known online news services to the political candidates themselves, citizens with an interest in politics can now obtain information from a myriad of sources, and are also able to engage in political discourse with many (often unknown) social media users and website commentators $[6,35,47,52]$. Although there has been an exponential increase in the information flow on online platforms, the human abilities to digest, analyze and process such information has been bounded due to biological brain constraints. It is argued that due to the bounded rationality theorem, when the humans have incomplete information about the alternatives, the probability of behaving irrationally is higher $[23,25,53]$. Therefore, social media users are generally unable to rationally analyze the abundant information flows on these emerging heterogeneous media.
People have a natural tendency to bond with those who are similar; a behaviour which is also imprinted in their selection of information sources and discussion groups. This principle, known as homophily, explains people's tendency to seek situations that imbue similarity and agreement; that is, people tend to bond with similar individuals $[2,9,34,37]$.

Because of the bounded rationality theorem and homophily, normal citizens interact with information sources and people who have similar beliefs during the selection process on social media services [5]. Thus, the widely accessible social media services turn potentially into breeding grounds of polarization. DiMaggio et al. [11] defined political polarization as the distance between the political orientations of different people. They argued that political polarization is a process as well as a state. While the latter refers to the distance an opinion is from some theoretical maximum or average, the former refers to development of the distance between the political orientations of different people over time.

DiMaggio et al. [11] introduced four independent and different polarization measurements, two of which referred to single distribution properties, while the others were focused on the relationships between the distributions. These measurements included variances or the dispersion of opinions, the kurtosis or bimodality of opinions, the tau-equivalent reliability or association between the opinions, and the correlation of opinions with salient individual characteristics. It was rationalized that political polarization would possibly entail a higher variance, a lower kurtosis, a higher tau-equivalent reliability and a higher correlation of opinions with salient individual characteristics.

\subsection{Current Research}

The motivation for creating reliable tools to measure and understand political polarization comes from political theory. In a democratic system, citizens should be aware of all cross-ideological points of view and also have the right to defend their own beliefs [22, 55]. Communication environments that expose citizens to a range of cross-ideological points allow citizens to be able to better develop justifications for their own viewpoints, establish a better understanding about alternative cross-ideological viewpoints, and develop a higher tolerance toward the opinions of others. DiMaggio et al. [11] claimed that "other things being equal, attitude polarization militates against social and political stability by reducing the probability of group formation at the center of the opinion distribution and by increasing the likelihood of the formation of groups with distinctive, irreconcilable policy preference". Therefore, as political polarization has been found to have undesirable effects, this paper seeks to develop a methodology to measure, analyze, and understand political polarization. Because online social media interactions are complex, a unique political polarization measurement is needed that is able to capture the dynamics or the evolution of political polarization over time.

This paper introduces social weighted bipartite endorsement networks, develops efficient methods to project weighted bipartite social networks that preserve the maximum amount of relevant information, and then applies the projection method to a simulated weighted bipartite social networks while controlling the political polarization. It is demonstrated that the search information index 
introduced by Trusina et al. [56] and Sneppen et al. [54] is positively correlated with the extent of the political polarization when applied to the projected networks. The newly developed methods are then applied to politically active Facebook network in Germany. The introduced measurements allow for the monitoring of the political polarization dynamics within social networks.

\section{RELATED WORK}

The relevant literature from two different topics is reviewed in this section; bipartite networks and political polarization.

\subsection{Projecting Bipartite Networks}

As mentioned, bipartite networks are applicable to many different fields of sciences. However, because of their inherent complexity, previous research has tended to only analyze their most basic features, such as the degree distribution of the vertices. There have been some attempts to introduce bipartite notion of local clustering coefficients [43, 50,59], centrality [14], correlation of vertex degree [46] and community detection $[21,57]$ that have been developed and directly applied to bipartite networks. However, as pointed out by Latapy et al. [29], as most of these measurements have been somewhat ad hoc and specific to the case, could not be easily extended to general bipartite networks.

The other approach to the study of bipartite networks is reducing the bipartite network to a binary or weighted one-mode network [3, 44, 61], with the most prominent projection methods being binary projection, sum projection, and the celebrated weighted sum projection of Newman [39]. Based on binary projection, two vertices of the same type are connected with a simple edge if both are at least connected to one vertex of the other type in the initial network. Under the sum projection, two vertices of the same type are connected with an edge weight $p$ if both are connected to $p$ vertices of the other type in the initial network. The Newman projection is similar to the sum projection except that each shared vertex of the other type is given a weight equal to $\frac{1}{N_{p}-1}$ when $N_{p}$ is the degree of that vertex.

Each projection method is based on a similarity function. For example, the binary projection is based on the following similarity function,

$$
\operatorname{Sim}_{\text {binary }}\left(u_{i}, u_{j}\right)= \begin{cases}1 & \text { if } u_{i} \cdot u_{j}>0 \\ 0 & \text { if } u_{i} \cdot u_{j}=0\end{cases}
$$

where $u_{i}$ is the binary vector indicating to which vertices the vertex $i$ is connected and - is the dot product. The sum method is based on the following similarity function

$$
\operatorname{Sim}_{\text {sum }}\left(u_{i}, u_{j}\right)=u_{i} \cdot u_{j}
$$

\subsection{Political Polarization Measurements}

DiMaggio et al. [11] introduced simple political polarization measurements that are not directly applicable to complex environment of social networks. Levendusky [32] attempted to measure and evaluate the polarization of individual Democrats and Republicans over time using National Election Study data. Fiorina and Abrams [15] studied the relationship between polarization and the geographical distribution of different groups. Freire [16] measured party polarization on the left-right scale. Using clustering methods, Conover et al. [7] showed that the network of political retweets had a segregated network of activity. Matakos et al. [33] used an opinion formation model to define a polarization index that measured the polarization in the opinions of the individuals in the network as well as the network structure. Akoglu [1] considered a bipartite network of users and subjects using Markovian Random Fields framework, and then defined the problem as a probabilistic classification task in which the polarity rank of the users in the political spectrum were to be predicted. The most related work to our methodology is Garimella et al. [17]. They used network theory tools to measure how controversial political topics in social media appeared to be.

\section{METHODOLOGY}

\subsection{Problem Overview}

The type of social networks which we considered are weighted bipartite social networks of users and discussion vertices. A bipartite network $\mathcal{G}=(\mathcal{U}, \mathcal{V}, \mathcal{E})$ was established in which $n$ users $\mathcal{U}=\left\{u_{1}, \ldots, u_{n}\right\}$ and $m$ discussions $\mathcal{V}=\left\{v_{1}, \ldots, v_{m}\right\}$ are connected with weighed edges $e(u, v, w) \in \mathcal{E}$ such that $\mathcal{E} \subseteq \mathcal{U} \times \mathcal{V} \times \mathbb{R}$. The weighted edges $e(u, v, w)$ represent positive endorsement of magnitude $w$ from user $u$ to discussion $v$. It was further assumed that each discussion $v$ belongs to one of $p>1$ possible non-empty classes $C_{V}=\left\{c_{1}, \ldots, c_{p}\right\}$ such that $p \ll m$; that is, each class $c$ is a sub-cluster of the network $\mathcal{G}$.

This structure can be applied to online user activities, such as the retweet and favorite networks on Twitter, the like and share networks on Facebook, and the share networks of blog posts. For example, set $\mathcal{C}$ might be $\{$ Republicans, Democrats $\}$ and set $\mathcal{V}$ might be a set of Facebook pages or blog pages that are politically oriented toward either Republicans or Democrats. Then, given the positive endorsement of $n$ users on the Facebook posts or blog pages, the task is to measure the ease of communication and also to capture dynamics of political polarization between the different network sub-clusters, \{Republicans, Democrats $\}$.

In the first step, tools were developed to project the weighted bipartite network $\mathcal{G}$ to the simple network $\mathcal{H}=(\mathcal{V}, \mathcal{V})$. To project $\mathcal{G}$ to $\mathcal{H}$, similar to other projection methods, a similarity function was needed. For the similarity function a distance function is employed and If the distance between two vertices was less than a maximum threshold, an edge between the two corresponding vertices was established. Then information theory concepts were applied to $\mathcal{H}$ to measure the ease of communication between every two random $\mathcal{H}$ vertices using the search information index introduced in Trusina et al. [56] and Sneppen et al. [54].

\subsection{Metric Function}

In this section, the distance or metric function is introduced that measures the similarity between the discussion vertices. Based on $e$ neighborhood graph construction method, if the distance between two vertices was less than a max threshold, they were seen to be similar vertices. Consider the adjacency matrix for $\mathcal{G}, A_{\mathcal{G}}$, in which each row represents a discussion vertex $v$ and each column a user vertex $u$. Let $S_{n}$ be the set of all permutations on $\mathcal{U}$, with each row of $A_{\mathcal{G}}$ being an element of $S_{n}$. For all $\sigma \in S_{n}$ define $\sigma(i)$ as 
the rank of the element $i \in \mathcal{U}$ in $\sigma$. For two elements $\sigma, \tau \in S_{n}$ the Kendall's tau $K(\sigma, \tau)$ is the initial metric introduced by Kendall [26]. Kendall's tau metric is identity invariant; that is the value of the metric does not depend on the actual identity of the elements in $\mathcal{U}$. Therefore, it suffices to consider $K(\sigma)=K(\sigma, 1)$ where 1 is the identity permutation. Then

$$
K(\sigma)=\sum_{(i, j): i>j} \mathbb{1}[\sigma(i)<\sigma(j)]
$$

where $\mathbb{1}$ is the indicator function. $K(\sigma)$ counts the total number of pairwise inversions between the elements of $\sigma$ and $\tau$.

In this study, one of the three new generalizations to the distance function introduced by Kumar and Vassilvitskii [28] was employed. The generalization aims to adjust the effect of swapping similar items. The intuition is that a pairwise inversion of two similar items should be penalized less than a pairwise inversion of two dissimilar items. Let $D$ be a non-empty metric on $\mathcal{U}$ and let $D_{i j}$ be the distance between users $i, j \in \mathcal{U}$. In this study, we defined the metric $D$ using the Jaccard index

$$
D\left(u_{i}, u_{j}\right)=1-J\left(u_{i}, u_{j}\right)=1-\frac{\left|P_{i} \cap P_{j}\right|}{\left|P_{i} \cup P_{j}\right|}
$$

where $P_{i}$ is a set consisting of the discussion vertices in which user $i$ has a non-zero endorsement on.

Then the similarity-adjusted distance between the rankings would be

$$
K^{*}(\sigma)=\sum_{(i, j): i<j} D_{i j} \mathbb{1}[\sigma(i)>\sigma(j)]
$$

$K^{*}(\sigma)$ as defined above was used to transform $\mathcal{G}$ by finding the distance between every two rows of $A_{\mathcal{G}}$. After transforming $\mathcal{G}$, $\mathcal{H}$ was defined based on the $e$-neighborhood graph construction method. In other words, $\mathcal{H}=(\mathcal{V}, \mathcal{V})$ was defined such that there would be an edge between two discussion vertices $v, v^{\prime} \in \mathcal{V}$ if the distance between $v$ and $v^{\prime}$ in the transformed $\mathcal{G}$ was smaller than $e \in \mathbb{R}^{+}$.

This similarity measurement preserves the local clustering in the initial bipartite network since it takes the similarity in users' behavior into consideration. If two users belong to the same local cluster, they would endorse similar discussion vertices. Therefore, the similarity measure $D\left(u_{i}, u_{j}\right)$ would be close to zero. This means that users of the same political orientation who lie within the same local cluster did not significantly affect the overall distance between two discussion vertices.

$\mathcal{H}$ is a simple one-mode network in which the vertices represent the discussion vertices. It inherits the classes of the discussion vertices from $\mathcal{G}$.

\subsection{Measurement of Accessibility Between Network Vertices}

The information flow between different vertices is only feasible through the local interactions between the adjacent vertices; therefore, close vertices are more accessible than distant vertices. The overall accessibility of the vertices or the reliability of the information transfer is thus a function of the network topology.

To measure the accessibility of vertices $v, v^{\prime} \in \mathcal{V}$ of $\mathcal{H}$, it was assumed that a bit of information is released from $v$ to $v^{\prime}$, which was then assumed to randomly traverse the network until it reaches $v^{\prime}$. Then, the probability of this bit of information traversing the shortest path is

$$
P\left\{p\left(v, v^{\prime}\right)\right\}=\frac{1}{k_{v}} \prod_{j \in p\left(v, v^{\prime}\right)} \frac{1}{k_{j}-1}
$$

where $p\left(v, v^{\prime}\right)$ is the shortest path between $v$ and $v^{\prime}, j$ is counting each vertex on the path, and $k_{j}$ is the degree of the vertex $j$. If some information is sent from $v$ to $v^{\prime}$ without the knowledge of the network map, then $P\left\{p\left(v, v^{\prime}\right)\right\}$ measures the probability that this information goes through the shortest path from $v$ and $v^{\prime}[54,56]$.

The search information index or the amount of the information needed to identify one of all the possible shortest paths between $v, v^{\prime}$ is defined as

$$
S\left(v \rightarrow v^{\prime}\right)=-\log _{2}\left(\sum_{p\left(v, v^{\prime}\right)} P\left\{p\left(v, v^{\prime}\right)\right\}\right)
$$

where the sum runs over all the shortest paths between $v$ and $v^{\prime}$. A high value for $S\left(v \rightarrow v^{\prime}\right)$ means that many yes/no questions are needed to locate $v^{\prime}$; therefore, a higher search information index between two vertices implies less availability of information between the vertices.

\subsection{Link Between the Search Information Index and Political Polarization}

DiMaggio et al. [11] defined political polarization as the distance between the political orientation of different people or "the extent of disagreement. [...] It is in the extremity of and distance between responses, not in their substantive content, that polarization inhere. [...] Polarization as a process refers to the increase in such opposition over time". As political polarization increases, it is expected that the users contribute more to the discussion vertices of a single political orientation. This leads to lower availability of information between each pair of discussion vertices with contrasting political orientation. Therefore, the average search information index between all possible pairs of discussion vertices with contrasting political orientation is expected to be higher.

The set $\mathcal{T}$ is defined as,

$$
\mathcal{T}_{c, c^{\prime}}=\left\{S\left(v \rightarrow v^{\prime}\right): \forall v, v^{\prime} \in \mathcal{V} \mid c_{v}=c, c_{v^{\prime}}=c^{\prime}\right\}
$$

where $c_{v}$ indicates the class of the discussion vertex $v$. We defined the polarization index between the two sub-clusters $c$ and $c^{\prime}$ as the average of the elements in set $\mathcal{T}$, which is named $\mathcal{P}_{\mathcal{T}} . \mathcal{P}_{\mathcal{T}}$ is expected to be increasing over time as the political polarization increases.

\section{RESULTS}

\subsection{Simulations}

Our ultimate goal of the simulations was to demonstrate that the search information index was highly correlated with the political polarization as introduced in DiMaggio et al. [11]. Based on DiMaggio et al., political polarization is a process that refers to the increase in the extent of disagreement over time. To simulate the polarization, two different parameter sets were defined: one that related to the distribution of endorsements when the political orientation of the user matched the political orientation of the discussion vertex, and the other that corresponded to the distribution of endorsements 


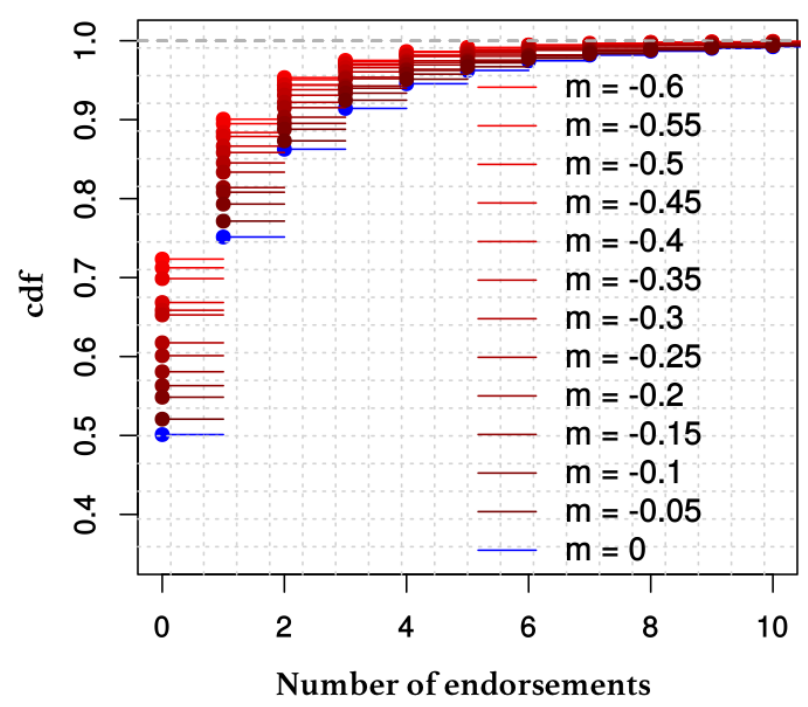

Figure 1: CDF of the endorsement distribution for different values of $m$.

when the orientation of the user and the orientation of the discussion vertex did not match. When the distance between these two distributions increases, the political polarization also increases.

To run the simulations, a two-class Facebook political sphere of Republicans and Democrats $\{r, d\}$ was assumed. Twenty Facebook political posts were simulated and in each run were randomly assigned to one of the parties. A pool of 800 users was created, and in each run, it was assumed that each user had a $50 \%$ probability of being Democratic or the Republican party supporter. $X_{\text {partisan,page }}$ was defined as the random variable for the number of likes a partisan gave to the Facebook posts of pages of a specific party. The following distribution for the number of likes a user contributes to Facebook posts with similar political orientation was also assumed; e.g., a Democrat on Democratic pages or a Republican on Republican pages:

$$
X_{p, p} \sim\lfloor\operatorname{lnorm}(0,1)\rfloor \text { for } p \in\{r, d\}
$$

where $\ln \operatorname{rom}(\mu, \sigma)$ stands for a log-normal distribution with a mean $\mu$ and standard deviation $\sigma$. The number of likes a user contributed to a Facebook page that had a contrasting political orientation, e.g., a Republican on a Democratic pages, was assumed to have the following distribution:

$$
X_{p, q}(m) \sim\lfloor\operatorname{lnorm}(m, 1)\rfloor \text { for } p \in\{r, d\}, p \neq q
$$

where $m \in\{-0.05,-0.10,-0.15,-0.20, \ldots,-0.6\}$.

As the value of $m$ decreases from 0 to -0.6 , the distance between the $X_{p, p}$ distribution and the $X_{p, q}(m)$ increases (Figure 1), which implies that as the value of $m$ decreases, the users contribute fewer endorsements to the vertices of the contrasting political inclination;

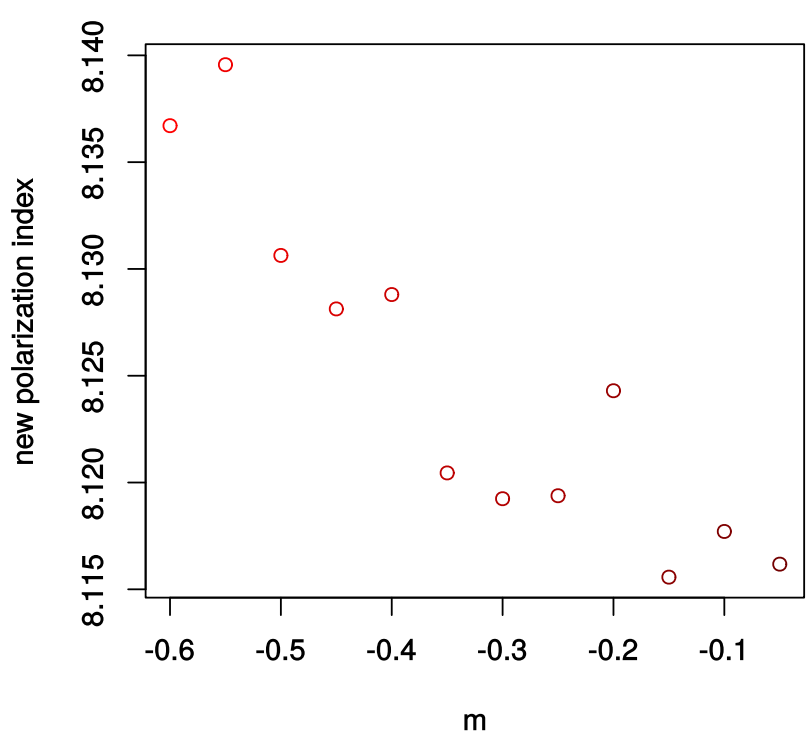

Figure 2: $\mathcal{P}_{\mathcal{T}}$ values against $m$.

therefore, based on the definition in DiMaggio et al. [11], political polarization increases as the value of $m$ decreases.

For each value of $m$, we ran our method on the simulated data for 1000 times and averaged the results. As can be seen in Figure 2 , it was confirmed that as the political polarization increased $(m$ decreases), as expected the value of the political polarization index $\mathcal{P}_{\mathcal{T}}$ also increased (with a correlation of 0.904 ).

\subsection{Facebook Data}

In this section, the proposed method was applied to Facebook data to determine whether the results agreed with the previous theoretical findings. Using Facebook's public API, all the posts on the official pages of all six active political parties in Germany (AfD, CDU, SPD, Die Linke, Grünen, and CSU) published in 2017 and all users who had endorsed these posts by making Facebook likes were downloaded. In total, 4,438,157 likes on 2,452 public Facebook posts were collected from 2,021,987 unique Facebook users. The data was then split into one-week windows and the bipartite network of user endorsements were constructed on the discussion vertices. It is important to notice that in this case the constructed network is a binary bipartite network but not weighted. This is because each user can like each Facebook post only for one time. Figure 3 shows the search information index between the AfD sub-cluster and all other sub-clusters, from which it can be seen that the average search information index between the AfD Facebook posts and the Facebook posts of the other parties was increasing over time. This implies that the AfD and non-AfD supporters had increased their endorsement activities on the pages connected to their own political orientation, and had decreased their activities on the pages connected to opposite political views. Therefore, these results could 
be seen as an increase in the levels of polarization between the AfD supporters and the other partisans. This is in line with previous studies on the rise of the AfD that found that the party adopted a political agenda that was quite different from the other parties, and consequently they had attracted alienated voters who had become segregated from the rest of the electorate [24, 31]. It was also found that the rise in party support had been accompanied by increased radicalization and polarization $[10,19,49]$.

\section{DISCUSSION}

In this paper, a new methodology for analyzing social networks was introduced that considered all the important properties of the structure of social networks such as associative mixing and local clustering. When the method was applied to political activity networks, it functioned as a proxy for the dynamics of political polarization; that is, the $\mathcal{P}_{\mathcal{T}}$ positively correlated to the level of political polarization in the network. The methodology was tested on both simulated data and user endorsement Facebook data from the German political party pages.

The development of this new method provides new insights for analyzing and understanding online political interactions. Social media service data can be used to evaluate theoretical social science questions, and this study provides a new tool to allow for this possibility. Given the multiplicity of social media data available today, researchers can use the newly proposed mathematical method to reveal the dynamics of political polarization. As this method does not discriminate endorsement types, it can be applied to different platforms. However, there are some limitations for the use of this method. While it can be used as a proxy for the dynamics of political polarization, it cannot directly provide insights as to the degree of polarization because the $\mathcal{P}_{\mathcal{T}}$ measure has no theoretical maximum value.

Similar to other online social network research, this study was dependent on the input data. Therefore, it is important to highlight the difficulties associated with extracting the proper data to ensure insightful scientific results. Unfortunately, there are often restrictions on the amount and type of data that can be acquired from social media platforms [45], and data quality is also a problem because of the level of bias [48]. Therefore, these features need to be considered in social media research and especially when studying important political processes.

The following further future directions are proposed:

- the application of the proposed method on case studies such as the polarization in U.S. online media , which appears extremely segregated [13].

- the extension of the method to define a theoretical maximum value of political polarization.

- the extension of the method to determine the discussion vertices that act as gatekeepers.

\section{REFERENCES}

[1] Leman Akoglu. 2014. Quantifying Political Polarity Based on Bipartite Opinion Networks. https://www.aaai.org/ocs/index.php/ICWSM/ICWSM14/paper/view/ $8073 / 8100$

[2] Delia Baldassarri and Peter Bearman. 2007. Dynamics of Political Polarization. American Sociological Review 72, 5 (2007), 784-811. https://doi.org/10.1177/ 000312240707200507 arXiv:https://doi.org/10.1177/000312240707200507
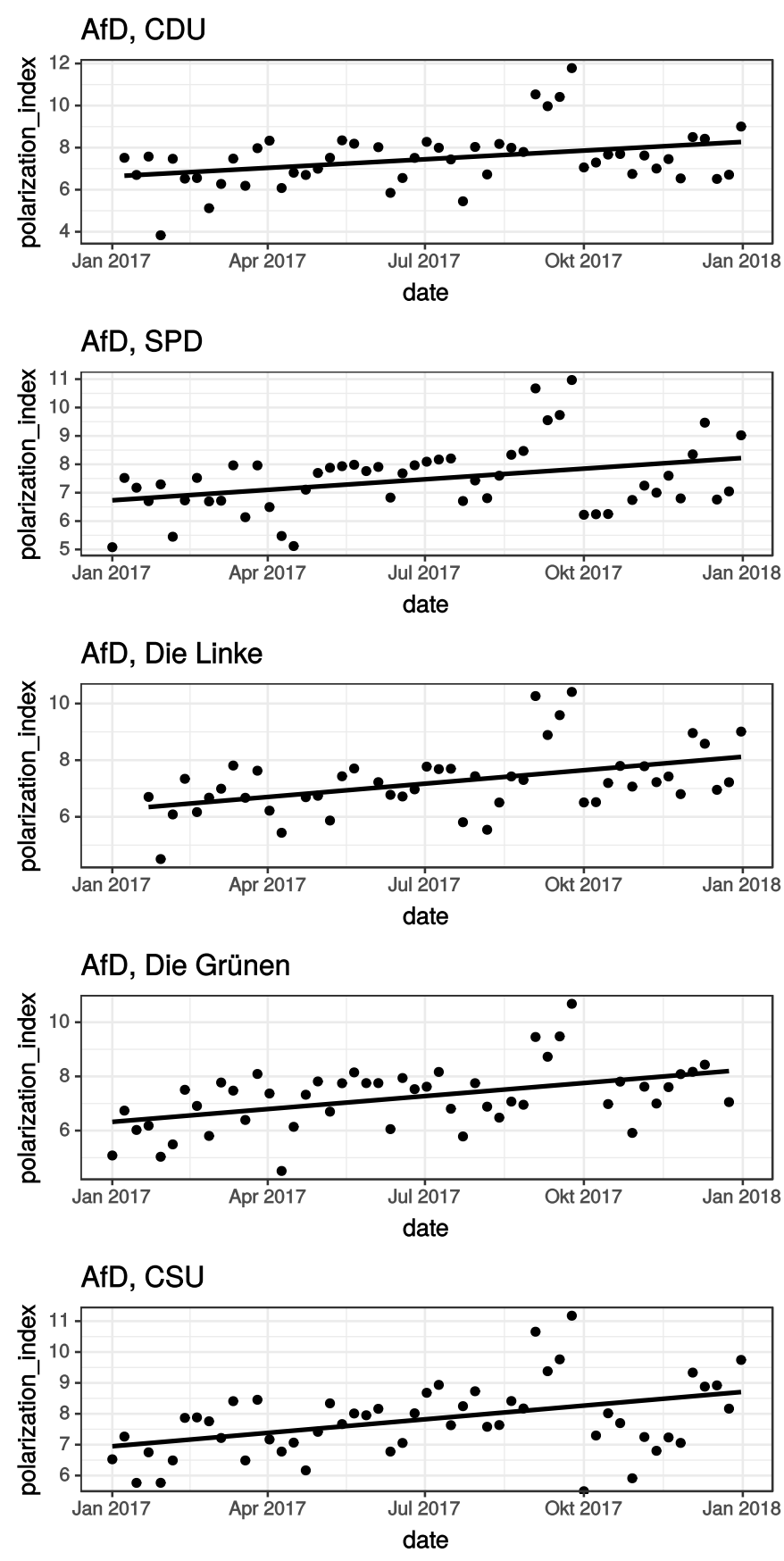

Figure $3: \mathcal{P}_{\mathcal{T}}$ weekly values for the party $A f D$

[3] S. Battiston and M. Catanzaro. 2004. Statistical properties of corporate board and director networks. The European Physical fournal B 38, 2 (March 2004), 345-352. https://doi.org/10.1140/epjb/e2004-00127-8

[4] N. Benchettara, R. Kanawati, and C. Rouveirol. 2010. Supervised Machine Learning Applied to Link Prediction in Bipartite Social Networks. In 2010 International Conference on Advances in Social Networks Analysis and Mining. 326-330. https://doi.org/10.1109/ASONAM.2010.87

[5] W. Lance Bennett. 2012. The Personalization of Politics: Political Identity, Social Media, and Changing Patterns of Participation. The ANNALS of the American Academy of Political and Social Science 644, 1 (2012), 20-39. https://doi.org/10. 
1177/0002716212451428 arXiv:https://doi.org/10.1177/0002716212451428

[6] W. Lance Bennett and Shanto Iyengar. 2008. A New Era of Minimal Effects? the Changing Foundations of Political Communication. Fournal of Communica tion 58, 4 (12 2008), 707-731. https://doi.org/10.1111/j.1460-2466.2008.00410.x arXiv:http://oup.prod.sis.lan/joc/article-pdf/58/4/707/22323968/jjnlcom0707.pdf

[7] Michael Conover, Jacob Ratkiewicz, Matthew Francisco, Bruno Goncalves, Filippo Menczer, and Alessandro Flammini. 2011. Political Polarization on Twitter. (2011) https://www.aaai.org/ocs/index.php/ICWSM/ICWSM11/paper/view/2847

[8] Wang Dahui, Zhou Li, and Di Zengru. 2006. Bipartite producer-consumer networks and the size distribution of firms. Physica A: Statistical Mechanics and its Applications 363, 2 (2006), 359-366. https://doi.org/10.1016/j.physa.2005.08.006

[9] Munmun De Choudhury, Hari Sundaram, Ajita John, Doree Duncan Seligmann, and Aisling Kelliher. 2010. "Birds of a Feather": Does User Homophily Impact Information Diffusion in Social Media? arXiv e-prints, Article arXiv:1006.1702 (June 2010), arXiv:1006.1702 pages. arXiv:cs.CY/1006.1702

[10] Frank Decker. 2016. The "Alternative for Germany:" Factors Behind its Emergence and Profile of a New Right-wing Populist Party. German Politics and Society 34, 2 (2016), 1-16. https://www.berghahnjournals.com/view/journals/gps/34/2/ gps340201.xml

[11] Paul DiMaggio, John Evans, and Bethany Bryson. 1996. Have American's Social Attitudes Become More Polarized? Amer. F. Sociology 102, 3 (1996), 690-755 https://doi.org/10.1086/230995 arXiv:https://doi.org/10.1086/230995

[12] M.G. Everett and S.P. Borgatti. 2013. The dual-projection approach for two-mode networks. Social Networks 35, 2 (2013), 204-210. https://doi.org/10.1016/j.socnet 2012.05.004 Special Issue on Advances in Two-mode Social Networks.

[13] Robert Faris, Hal Roberts, Bruce Etling, Nikki Bourassa, Ethan Zuckerman, and Yochai Benkler. 2017. Partisanship, propaganda, and disinformation: Online media and the 2016 US presidential election. (2017).

[14] Katherine Faust. 1997. Centrality in affiliation networks. Social Networks 19, 2 (1997), 157-191. https://doi.org/10.1016/S0378-8733(96)00300-0

[15] Morris P. Fiorina and Samuel J. Abrams. 2008. Political Polarization in the American Public. Annual Review of Political Science 11, 1 (2008), 563-588. https://doi.org/10.1146/annurev.polisci.11.053106.153836 arXiv:https://doi.org/10.1146/annurev.polisci.11.053106.153836

[16] André Freire. 2008. Party Polarization and Citizens' Left-Right Orientations. Party Politics 14, 2 (2008), 189-209. https://doi.org/10.1177/1354068807085889 arXiv:https://doi.org/10.1177/1354068807085889

[17] Kiran Garimella, Gianmarco De Francisci Morales, Aristides Gionis, and Michael Mathioudakis. 2018. Quantifying Controversy on Social Media. Trans. Soc Comput. 1, 1, Article 3 (Jan. 2018), 27 pages. https://doi.org/10.1145/3140565

[18] Daniel Geschke, Jan Lorenz, and Peter Holtz. 2019. The triple-filter bub ble: Using agent-based modelling to test a meta-theoretical framework for the emergence of filter bubbles and echo chambers. British fournal of Social Psychology 58, 1 (2019), 129-149. https://doi.org/10.1111/bjso.12286 arXiv:https://onlinelibrary.wiley.com/doi/pdf/10.1111/bjso.12286

[19] Karsten Grabow. 2016. Pegida and the Alternative FüR Deutschland: Two Sides of the Same Coin? European View 15, 2 (2016), 173-181. https://doi.org/10.1007/ s12290-016-0419-1 arXiv:https://doi.org/10.1007/s12290-016-0419-1

[20] Jean-Loup Guillaume, Matthieu Latapy, and Stevens Le-Blond. 2005. Statistical Analysis of a P2P Query Graph Based on Degrees and Their Time-Evolution. In Distributed Computing - IWDC 2004, Arunabha Sen, Nabanita Das, Sajal K. Das, and Bhabani P. Sinha (Eds.). Springer Berlin Heidelberg, Berlin, Heidelberg, 126-137.

[21] Roger Guimerà, Marta Sales-Pardo, and Luís A. Nunes Amaral. 2007. Module identification in bipartite and directed networks. Phys. Rev. E 76 (Sept. 2007), 36102. Issue 3. https://doi.org/10.1103/PhysRevE.76.036102

[22] David Held. 2006. Models of democracy. Stanford University Press.

[23] Martin Hilbert. 2011. Toward a Synthesis of Cognitive Biases: How Noisy Information Processing Can Bias Human Decision Making. Psychological bulletin 138 (11 2011), 211-37. https://doi.org/10.1037/a0025940

[24] Michael Jankowski, Sebastian Schneider, and Markus Tepe. 2017. Ideological alternative? Analyzing Alternative für Deutschland candidates' ideal points via black box scaling. Party Politics 23, 6 (2017), 704-716. https://doi.org/10.1177/ 1354068815625230 arXiv:https://doi.org/10.1177/1354068815625230

[25] Bryan D. Jones. 1999. BOUNDED RATIONALITY. Annual Review of Political Science 2, 1 (1999), 297-321. https://doi.org/10.1146/annurev.polisci.2.1.297 arXiv:https://doi.org/10.1146/annurev.polisci.2.1.297

[26] Maurice G. Kendall. 1948. Rank correlation methods. (1948) http://gso.gbv.de/DB=2.1/CMD?ACT=SRCHA\&SRT=YOP \&IKT=1016\&TRM= ppn+18489199X\&sourceid=fbw_bibsonomy

[27] Gary King. 2014. Restructuring the Social Sciences: Reflections from Harvard's Institute for Quantitative Social Science. PS: Political Science \& Politics 47, 1 (2014), 165-172. https://doi.org/10.1017/S1049096513001534

[28] Ravi Kumar and Sergei Vassilvitskii. 2010. Generalized Distances Between Rankings. In Proceedings of the 19th International Conference on World Wide Web (WWW '10). ACM, New York, NY, USA, 571-580. https://doi.org/10.1145/1772690.1772749

[29] Matthieu Latapy, Clémence Magnien, and Nathalie Del Vecchio. 2008. Basic notions for the analysis of large two-mode networks. Social Networks 30, 1 (2008)
31-48. https://doi.org/10.1016/j.socnet.2007.04.006

[30] Stevens Le Blond, Jean-Loup Guillaume, and Matthieu Latapy. 2005. Clustering in P2P Exchanges and Consequences on Performances. In Peer-to-Peer Systems $I V$, Miguel Castro and Robbert van Renesse (Eds.). Springer Berlin Heidelberg, Berlin, Heidelberg, 193-204.

[31] Charles Lees. 2018. The 'Alternative for Germany': The rise of right-wing populism at the heart of Europe. Politics 38, 3 (2018), 295-310. https://doi.org/10. 1177/0263395718777718 arXiv:https://doi.org/10.1177/0263395718777718

[32] Matthew S. Levendusky. 2009. The Microfoundations of Mass Polarization. Political Analysis 17, 2 (2009), 162-176. https://doi.org/10.1093/pan/mpp003

[33] Antonis Matakos, Evimaria Terzi, and Panayiotis Tsaparas. 2017. Measuring and moderating opinion polarization in social networks. Data Mining and Knowledge Discovery 31, 5 (Sept. 2017), 1480-1505. https://doi.org/10.1007/ s10618-017-0527-9

[34] J. Miller McPherson and Lynn Smith-Lovin. 1987. Homophily in Voluntary Organizations: Status Distance and the Composition of Face-to-Face Groups. American Sociological Review 52, 3 (1987), 370-379. http://www.jstor.org/stable/ 2095356

[35] A Mitchell and R Weisel. 2014. Political polarization \& media habits: From Fox News to Facebook, how liberals, and conservatives keep up with politics. Pew Research Center. (2014)

[36] Peter J. Mucha, Thomas Richardson, Kevin Macon, Mason A. Porter, and Jukka-Pekka Onnela. 2010. Community Structure in Time-Dependent, Multiscale, and Multiplex Networks. Science $328, \quad 5980$ (2010), 876-878. https://doi.org/10.1126/science.1184819 arXiv:https://science.sciencemag.org/content/328/5980/876.full.pdf

[37] Jonathan Mummolo and Clayton Nall. 2017. Why Partisans Do Not Sort: The Constraints on Political Segregation. The fournal of Politics 79, 1 (2017), 45-59. https://doi.org/10.1086/687569 arXiv:https://doi.org/10.1086/687569

[38] Mark E.J. Newman. 2004. Who Is the Best Connected Scientist?A Study of Scientific Coauthorship Networks. In Complex Networks, Eli Ben-Naim, Hans Frauenfelder, and Zoltan Toroczkai (Eds.). Springer Berlin Heidelberg, Berlin, Heidelberg, 337-370. https://doi.org/10.1007/978-3-540-44485-5 16

[39] M. E. J. Newman. 2001. Scientific collaboration networks. II. Shortest paths, weighted networks, and centrality. Phys. Rev. E 64 (June 2001), 16132. Issue 1. https://doi.org/10.1103/PhysRevE.64.016132

[40] M. E. J. Newman and Juyong Park. 2003. Why social networks are different from other types of networks. Phys. Rev. E 68 (Sept. 2003), 36122. Issue 3. https://doi.org/10.1103/PhysRevE.68.036122

[41] M. E. J. Newman, S. H. Strogatz, and D. J. Watts. 2001. Random graphs with arbitrary degree distributions and their applications. Phys. Rev. E 64 (July 2001), 26118. Issue 2. https://doi.org/10.1103/PhysRevE.64.026118

[42] Roberto N. Onody and Paulo A. de Castro. 2004. Complex network study of Brazilian soccer players. Phys. Rev. E 70 (Sept. 2004), 37103. Issue 3. https: //doi.org/10.1103/PhysRevE.70.037103

[43] Tore Opsahl. 2013. Triadic closure in two-mode networks: Redefining the global and local clustering coefficients. Social Networks 35, 2 (2013), 159-167. https: //doi.org/10.1016/j.socnet.2011.07.001 Special Issue on Advances in Two-mode Social Networks.

[44] Benigno Padrón, Manuel Nogales, and Anna Traveset. 2011. Alternative approaches of transforming bimodal into unimodal mutualistic networks. The usefulness of preserving weighted information. Basic and Applied Ecology 12, 8 (2011), 713-721. https://doi.org/10.1016/j.baae.2011.09.004

[45] Orestis Papakyriakopoulos, Simon Hegelich, Morteza Shahrezaye, and Juan Carlos Medina Serrano. 2018. Social media and microtargeting: Political data processing and the consequences for Germany. Big Data \& Society 5,2 (2018), 2053951718811844. https://doi.org/10.1177/2053951718811844 arXiv:https://doi.org/10.1177/2053951718811844

[46] Matti Peltomäki and Mikko Alava. 2006. Correlations in bipartite collaboration networks. Fournal of Statistical Mechanics: Theory and Experiment 2006, 1 (Jan. 2006), P01010-P01010. https://doi.org/10.1088/1742-5468/2006/01/p01010

[47] Iryna Pentina and Monideepa Tarafdar. 2014. From "information" to "knowing": Exploring the role of social media in contemporary news consumption. Computers in Human Behavior 35 (2014), 211-223. https://doi.org/10.1016/j.chb.2014.02.045

[48] Jürgen Pfeffer, Katja Mayer, and Fred Morstatter. 2018. Tampering with Twitter's Sample API. EPf Data Science 7, 1 (Dec. 2018), 50. https://doi.org/10.1140/epjds/ s13688-018-0178-0

[49] Thea Riebe, Katja Pätsch, Marc-André Kaufhold, and Christian Reuter. 2018. From Conspiracies to Insults: A Case Study of Radicalisation in Social Media Discourse. In Mensch und Computer 2018 - Workshopband, Raimund Dachselt and Gerhard Weber (Eds.). Gesellschaft für Informatik e.V., Bonn.

[50] Garry Robins and Malcolm Alexander. 2004. Small Worlds Among Interlocking Directors: Network Structure and Distance in Bipartite Graphs. Computational \& Mathematical Organization Theory 10, 1 (May 2004), 69-94. https://doi.org/10. 1023/B:CMOT.0000032580.12184.c0

[51] Camille Roth and Paul Bourgine. 2005. Epistemic Communities: Description and Hierarchic Categorization. Mathematical Population Studies 12, 2 (2005), 107-130. https://doi.org/10.1080/08898480590931404 
arXiv:https://doi.org/10.1080/08898480590931404

[52] Juan Carlos Medina Serrano, Simon Hegelich, Morteza Shahrezaye, and Orestis Papakyriakopoulos. 2018. Social Media Report: The 2017 German Federal Elections (1 ed.). TUM.University Press.

[53] Herbert A Simon. 1972. Theories of bounded rationality. Decision and organization 1, 1 (1972), 161-176

[54] K Sneppen, A Trusina, and M Rosvall. 2005. Hide-and-seek on complex networks Europhysics Letters (EPL) 69, 5 (March 2005), 853-859. https://doi.org/10.1209/ epl/i2004-10422-0

[55] Cass R. Sunstein. 2002. The Law of Group Polarization. Journal of Political Philosophy 10, 2 (2002), 175-195. https://doi.org/10.1111/1467-9760.00148 arXiv:https://onlinelibrary.wiley.com/doi/pdf/10.1111/1467-9760.00148

[56] A. Trusina, M. Rosvall, and K. Sneppen. 2005. Communication Boundaries in Networks. Phys. Rev. Lett. 94 (June 2005), 238701. Issue 23. https://doi.org/10. 1103/PhysRevLett.94.238701

[57] J. Yang and J. Leskovec. 2012. Community-Affiliation Graph Model for Overlapping Network Community Detection. In 2012 IEEE 12th International Conference on Data Mining. 1170-1175. https://doi.org/10.1109/ICDM.2012.139

[58] Jaewon Yang, Julian McAuley, and Jure Leskovec. 2014. Detecting Cohesive and 2-mode Communities Indirected and Undirected Networks. In Proceedings of the 7th ACM International Conference on Web Search and Data Mining (WSDM '14). ACM, New York, NY, USA, 323-332. https://doi.org/10.1145/2556195.2556243

[59] Peng Zhang, Jinliang Wang, Xiaojia Li, Menghui Li, Zengru Di, and Ying Fan. 2008. Clustering coefficient and community structure of bipartite networks. Physica A: Statistical Mechanics and its Applications 387, 27 (2008), 6869-6875. https://doi.org/10.1016/j.physa.2008.09.006

[60] K. Zhao, X. Wang, M. Yu, and B. Gao. 2014. User Recommendations in Reciprocal and Bipartite Social Networks-An Online Dating Case Study. IEEE Intelligent Systems 29, 2 (March 2014), 27-35. https://doi.org/10.1109/MIS.2013.104

[61] Tao Zhou, Jie Ren, Matú š Medo, and Yi-Cheng Zhang. 2007. Bipartite network projection and personal recommendation. Phys. Rev. E 76 (Oct. 2007), 46115. Issue 4. https://doi.org/10.1103/PhysRevE.76.046115 